TERRA. Revista de Desarrollo Local e-ISSN: 2386-9968

Número 7 (2020), 322-328

DOI 10.7203/terra.7.19146

IIDL - Instituto Interuniversitario de Desarrollo Local

\title{
Reseña. Áreas metropolitanas. Una descripción a partir de la complejidad sistémica
}

\author{
Carolina Burriel Hernández \\ Graduada en Geografía y Medio Ambiente (Valencia, España) y Máster en \\ Planificación y Desarrollo Territorial Sostenible (Madrid, España) \\ carolina.burriel@estudiante.uam.es
}

Esta obra se distribuye con la licencia Creative Commons Reconocimiento-NoComercial-SinObraDerivada 4.0 Internacional 


\section{SECCIÓN RESEÑAS}

\section{Reseña. Áreas metropolitanas. Una descripción a partir de la complejidad sistémica}

Resumen. La reciente obra de Josep Pont Vidal cuenta como las áreas metropolitanas poseen características que les permiten abordar problemáticas complejas tales como las de corte ambiental, económico, social y/o político. En ocasiones, las áreas metropolitanas se encuentran inmersas en desafíos que los modelos organizativos tradicionales, basados en el Estado, regiones y municipios no pueden determinar, y que demandan el amparo de nuevos paradigmas teóricos. La principal aportación de la obra que aquí reseñamos, es el análisis de la (hiper)complejidad, característica de las sociedades actuales, así como la capacidad de adecuación autoorganizativa de las áreas metropolitanas. Este proceso se debe de producir por medio de operaciones que actúan de forma convergente circular (interna y externa), a partir de la interacción comunitaria entre sistema y entorno. A modo de conclusión, el autor explica que la gobernanza se encuentra hoy por hoy en una situación marcada por la crisis. En este panorama caracterizado por la inseguridad, el riesgo y la complejidad, entiende que es necesario organizar un debate teórico conceptual de la gobernanza urbana y metropolitana. Desgraciadamente, seguimos encontrándonos en una época de ignorancia colectiva, donde la transformación solo tendrá lugar cuando la sociedad pueda tener las consecuencias de sus efectos.

Palabras clave. Teoría de la complejidad, gobernanza, auto-organización, autopoiesis.

Recibido: 01 de diciembre de 2020

Devuelto para revisión: -

Aceptado: 02 de diciembre de 2020

Referencia / Citation:

Burriel, C. (2020). Reseña. Áreas metropolitanas. Una descripción a partir de la complejidad sistémica. TERRA. Revista de Desarrollo Local, (7), 322-328. DOI 10.7203/terra.7.19146 


\section{ÁREAS METROPOLITANAS. UNA DESCRIPCIÓN A PARTIR DE LA COMPLEJIDAD SISTÉMICA}

Madrid, Editorial Sicómoro, 2019 (1era edición), 222 páginas

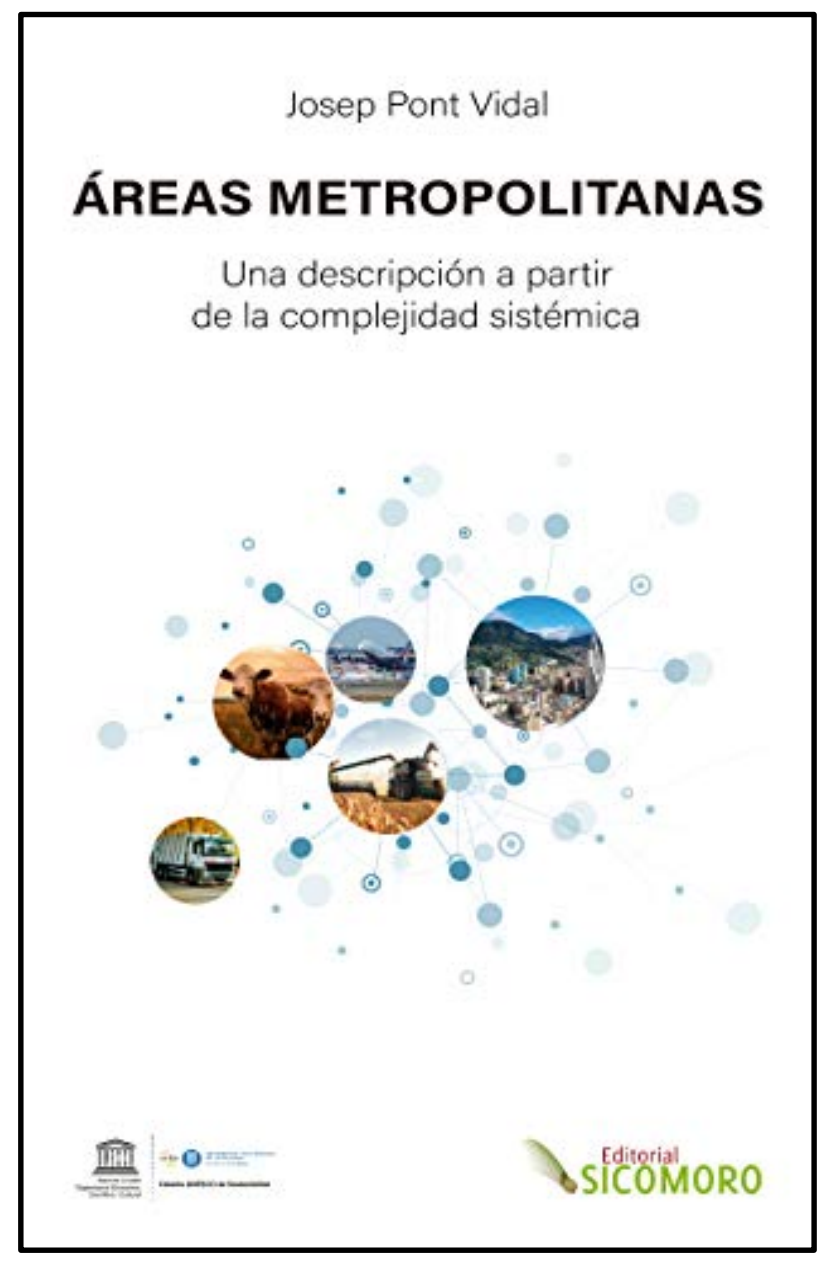

La reciente obra de Josep Pont Vidal cuenta como las áreas metropolitanas poseen características que les permiten abordar problemáticas complejas tales como: ambientales, económicas, sociales y políticas. En ocasiones, las áreas metropolitanas se encuentran inmersas en desafíos que los modelos organizativos tradicionales, basados en el Estado, regiones y municipios no pueden determinar, y que demandan el amparo de nuevos paradigmas teóricos.

La principal aportación de la obra que aquí reseñamos, es el análisis de la (hiper) complejidad, característica de las sociedades actuales, así como la capacidad de adecuación autoorganizativa de las áreas metropolitanas. Este proceso se debe de producir por medio de operaciones que actúan de forma convergente circular (interna y externa), a partir de la interacción comunitaria entre sistema y entorno.

El libro consta de cinco capítulos que describen el fenómeno metropolitano fundamentado en la Teoría de Sistemas Autorreferentes de Niklas Luhmann (Figura 1). No obstante, previo a estos encontramos un capítulo introductorio, en el cual se enmarcan la Teoría de Sistemas Autorreferentes, las áreas metropolitanas y sus procesos decisionales.

Una de las primeras aportaciones que se realizan, aborda la teoría de la complejidad para mostrar tanto las limitaciones como las posibilidades comunicativas de los sistemas, y se ejemplifica con los modelos teóricos metropolitanos existentes, quedando definidos por el autor como de carácter limitado, conceptual y observacional.

El primer capítulo, “Áreas metropolitanas y complejidad”, señala que el binomio conceptual compuesto por el área metropolitana y la gobernanza, todavía es ambiguo y permanece cuasi-inexplorado en los planos teórico, normativo y práctico. El término gobernanza no tiene una definición clara. De este modo, para poder definirla se utiliza un amplio abanico de fenómenos organizativos, administrativos, políticos y económicos.

Respecto al término área metropolitana se han realizado diferentes análisis que inciden en el plano político-administrativo, en el que se ha vivido un abandono de la cuestión 
metropolitana y se reclama la necesidad de un nuevo impulso. Además, en la perspectiva jurídica, escasos países de la Unión Europea disponen de la legislación específica para el reconocimiento del fenómeno supramunicipal, factor clave para la creación jurídica de entes metropolitanos ${ }^{1}$.

Otra de las cuestiones es la complejidad como nexo analítico, donde esta queda definida como un indicador de la arquitectura de las relaciones existentes entre las partes de un sistema, y a su vez, entre el sistema y su entorno. Esta relación enlaza la complejidad con el ámbito urbano, es decir, los elementos urbanos tienen relaciones complejas cuando poseen limitaciones internas. Por tanto, no es posible que un elemento se relacione en cada momento con otro elemento.

Figura 1. Índice de contenidos

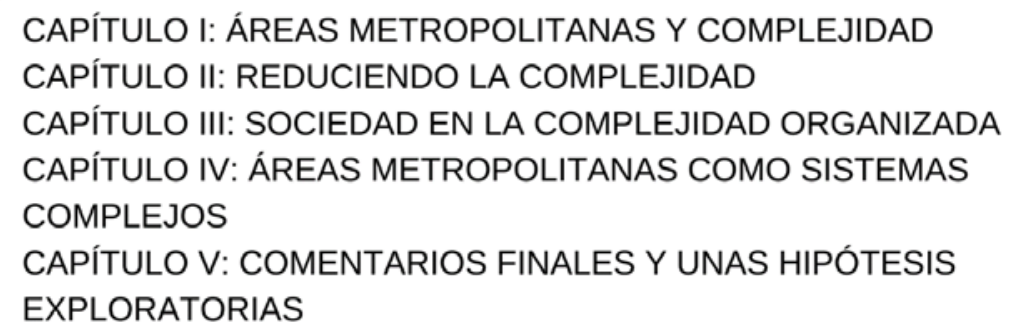

Fuente: elaboración propia a partir del índice de contenidos.

El segundo capítulo,"Reduciendo la complejidad”, nos adentra a conocer cómo ha ido evolucionando el término de complejidad. En el siglo XVIII, comenzó a ser aplicado en la fisiología y posteriormente se extendió a la economía, la química, la biología y la geometría. En las primeras décadas del siglo XX, el término era utilizado en el psicoanálisis, la psicología y la medicina. Ya en el siglo actual, Edgar Morin explicaría en su obra "La epistemología de la Complejidad" (2005), el "paradigma de la complejidad" concepto que se contrapone al "paradigma de la simplicidad”.

Entendemos por complejidad al conjunto de niveles que comprenden el plano ontológico, las formas de construcción del conocimiento y las metodologías y técnicas empleadas por el paradigma de la complejidad. Este término ha sido acuñado por las Ciencias Sociales, la Administración y la Gestión Pública. Pero, cabe destacar las dificultades en los campos de la Gestión y la Gobernanza, debido a que su complejidad es vista como un problema que debe de ser solucionado presentando la situación desde la simplicidad. Para ello, el autor describe la teoría de la complejidad organizada en cuatro planos: políticoadministrativo, ontológico, metodológico y técnico.

\footnotetext{
${ }^{1}$ Quizás una de las más recientes excepciones sea la próxima Ley de las Áreas Metropolitanas de la Comunidad Valenciana, elaborada por el Prof. del Dpto. de Geografía de la Universidad de Valencia, Joan Romero (https://www.uv.es/uvweb/departamento-geografia/es/novedades-del-departamento/catedraticojuan-romero-encargado-del-borrado-ley-areas-metropolitanas-comunitat-valenciana1285923518578/Novetat.html?id=1286154606383). De hecho, el Prof. Joan Romero ha guiado un reciente seminario internacional sobre esta temática (desarrollado on-line los días 23 y 24 de octubre de 2020), cuyos materiales estás disponibles en la web de la Cátedra que dirige, la Cátedra PROSPECT 2030: https://prospectcv2030.com/activities/gobernanza-de-las-areas-metropolitanas-en-europa/ (recuperado el 02 de diciembre de 2020).
} 
La complejidad organizada aparece cuando se define la complejidad como un estado de cosas auto-condicionado, donde una serie de elementos se organizan para construir una unidad superior enfocada a satisfacer el objetivo general. Mientras que la complejidad desorganizada, abarca la multiplicidad de fenómenos y las variables vinculadas a la economía, la política y al medioambiente. Este fenómeno se ejemplifica en las sociedades, donde se expone que las crisis económicas y sus impactos sociales pueden formular el diagnóstico de un tipo de sociedad compleja desorganizada.

Por consiguiente, se introduce el tema sobre la auto-organización de los sistemas, que será explicada en los siguientes capítulos. Dentro de las premisas principales en las que se sustenta la autoorganización de los sistemas complejos, existen dos tipos de aproximaciones: la primera, que proceden de centros de investigación básica, y la segunda, que provienen de estudios dirigidos para ser aplicados en el ámbito privado o empresarial. Ambos enfoques comparten conceptos centrales que conforman la idea de autoorganización de un sistema.

El tercer capítulo, "Sociedad y complejidad (Des-)organizada”, describe y expone el concepto de autorreferencialidad o autopoiesis, y su transición a la teoría de sistemas autorreferenciales. La transposición del término autopoiesis a la teoría de sistemas sociales se experimentó en la década de los años 80 por Luhmann, el cual recurrió a la descripción husserliana de sentido. Seguidamente, lo limitó a los sistemas sociales y psíquicos, con los que le atribuyó operaciones autorreferenciales en las que el "sentido" es un producto de las operaciones internas del sistema. También, se expone la hipótesis sobre si el carácter autorreferencial de un sistema impide que sea gobernado o influenciado desde el exterior, ya que se entiende que un sistema autopoiético es un sistema operativamente cerrado.

Otros de los autores que plantean posibilidades de autoorganización sistémica en el contexto técnico y social son Loet Leydesdorff (2015) e Ismael (2010). El primero mantiene la autoorganización como hipótesis, ya que cabría la posibilidad de la existencia de sistemas autoorganizados. Por su parte, el segundo describe el éxito de los modelos autoorganizados en fenómenos sociales como las “multitudes inteligentes". Posteriormente, se plantea la hipótesis de John-Steward Gordon sobre si una sociedad humana está limitada a no aceptar la autoorganización, al tender la última a producir resultados acordes con los objetivos generales de la sociedad.

En cuanto a la perspectiva de los sistemas autorreferenciales formulada por Luhmann, en la obra reseñada se trasciende el marco ontológico y antropológico clásico, donde se suele colocar al ser humano como centro de la investigación, situándose este en la periferia del sistema. Además, los procesos de autoorganización son definidos a partir de procesos internos de los sistemas y de conceptos como "clausura operativa", "autopoiesis" o "doble contingencia”. En concreto, cuando aparecen estos puntos de vista teóricos nos situamos en un periodo de confianza en el crecimiento económico y la racionalidad humana.

Seguidamente, se citan las diferencias entre la autoorganización institucional y la noinstitucional a partir de los marcos teóricos relativos a la Teoría de sistemas autorreferenciales y la Teoría neo-sistémica contextual. Después de ver la autoorganización en el plano de los sistemas complejos y en el plano meta-sociológico de la teoría de sistemas, se plantea transferir estos supuestos a la teoría del actor de Etzioni. A partir de aquí, se analizan las siguientes cuestiones: i) la propuesta de fusión entre las instituciones locales y regionales para lograr una mayor eficiencia; ii) la concepción sobre el carácter necesario de un equilibrio entre el Estado-comunidad-mercado; y iii) la idealización del individuo racional, orientado al bien común y a la comunidad. 
El cuarto capítulo da lugar a las “Áreas Metropolitanas como sistemas complejos”. Una vez analizadas estas desde el plano teórico y abstracto, los procesos de contingencia, la complejidad y la auto-organización, el autor nos explica cómo actúa la administración de un área metropolitana. Las administraciones municipales y las áreas metropolitanas se caracterizan por la diversidad de forma organizativa, la complejidad en la toma de decisiones y el dinamismo de sus estructuras. De ahí, sus decisiones, contingencias y riesgos.

Un área metropolitana se encuentra sujeta por una diferenciación formal entre sistema y entorno, al poseer límites jurisdiccionales referentes al territorio (conjunto de municipios). Este sistema que denominamos área metropolitana cuenta con un entorno formado por otros entes públicos como municipios, comarcas, consorcio y el Estado. Según Luhmann, estos entes públicos emiten diferentes tipos de comunicaciones en el sistema (área metropolitana), que se pueden manifestar como irritaciones o comunicaciones, las cuales se transmiten al área metropolitana en normativas y leyes emitidas por el gobierno regional o por el Estado.

Desde las últimas décadas del siglo XX, los gobiernos supramunicipales han aplicado modelos de organización, gestión y administración, clasificándose en cuatro corrientes conceptuales: Escuela de la reforma (Reform School), Elección racional, el Nuevo regionalismo, y la Sistémica o ambiental (sistemas abiertos).

La Escuela de la Reforma se inició a mediados de siglo XX y mantuvo la necesidad de poseer instrumentos de coordinación metropolitana, aunque limitando sus competencias. En cuanto a la teoría de la elección pública, mantiene la hipótesis de orientarse y favorecer la fragmentación o desagregación de las áreas metropolitanas como entes supramunicipales, garantizando mayores niveles de eficiencia y democracia. El Nuevo Regionalismo ha sido aplicado en diversas áreas metropolitanas inicialmente en ciudades norteamericanas como San Francisco, y a partir del siglo XXI, en ciudades europeas como Barcelona. Esta corriente, se basa en una nueva política de "escala” en la que las instituciones, los actores locales, y las instituciones sociales locales, se adaptan a los diversos procesos de reestructuración derivados de los efectos de la globalización. Por último, encontramos la corriente Sistémica o ambiental, la cual tiene su origen en la década de los setenta, donde establece un nexo urbano entre la Sociología, la Psicología, la Administración, el Diseño Urbano, la Ecología y el Urbanismo. Esta corriente, da por sentada la conexión interdisciplinaria y el pensamiento sistémico complejo, aproximándose a la teoría de sistemas.

En cuanto a óptica autorreferencial, no hay dudas de que en la sociedad existen sistemas, y que son necesarios para reducir la complejidad y supercomplejidad como se pone de manifiesto en las actuales sociedades modernas. No obstante, la reflexión sobre la existencia de sistemas no es exclusiva de Luhmann, ya que otros autores lo han definido con otros nombres como: superestructura e infraestructura (Marx), sistema y mundo de vida (Habermas), habitus y campo (Bourdieu), organización y comunidad (Etzioni), o esfera pública y privada (Koselleck).

El quinto capítulo, "Comentarios finales y unas hipótesis exploratorias", expone los principales desafíos que el autor de la obra aborda a lo largo de la misma. Son aquellos que hacen frente a las áreas metropolitanas en los ámbitos conceptuales, teóricos y organizativos, vinculándola con el territorio y su entorno. También, se han plasmado diferentes propuestas que estudian los sistemas complejos, y que aportan reflexiones para comprender las áreas metropolitanas desde un enfoque basado en la complejidad. El autor, nos explica que las aportaciones de la teoría de sistemas autorreferenciales de 
Luhmann no es una simple transposición, sino que se trata de una aportación sofisticada para la compresión de la hipercomplejidad de las sociedades modernas. Y, que ninguna teoría posee la verdad absoluta e indiscutible, ya que son meramente instrumentos de estudio para describir y analizar el mundo y los fenómenos de una forma clara.

A partir de la teoría de sistemas autorreferenciales de Luhmann se han descrito las áreas metropolitanas como un sistema autónomo y autorreferencial, ya que desarrolla sus operaciones internas y externas. También, se ha permitido describirlas y analizarlas desde la perspectiva empírica, dando origen a otro tipo de descripciones conceptuales y analíticas que permite estudiar las operaciones que realizan este tipo de entes públicos.

A modo de conclusión, el autor explica que la gobernanza se encuentra hoy por hoy en una situación marcada por la crisis. En este panorama caracterizado por la inseguridad, el riesgo y la complejidad, entiende que es necesario organizar un debate teórico conceptual de la gobernanza urbana y metropolitana. Desgraciadamente, seguimos encontrándonos en una época de ignorancia colectiva, donde la transformación solo tendrá lugar cuando la sociedad pueda tener las consecuencias de sus efectos.

Carolina Burriel Hernández Graduada en Geografía y Medio Ambiente, Universitat de València Máster en Planificación y Desarrollo Territorial Sostenible, Universidad Autónoma de Madrid 